\title{
Holography for the very early universe and the classic puzzles of hot big bang cosmology
}

\author{
Horatiu Nastase ${ }^{1, *}$ and Kostas Skenderis ${ }^{2, \dagger}$ \\ ${ }^{1}$ Instituto de Física Teórica, UNESP-Universidade Estadual Paulista, \\ Rua Dr. Bento T. Ferraz 271, Bl. II, São Paulo 01140-070, SP, Brazil \\ ${ }^{2}$ Southampton Theory Astrophysics Gravity (STAG) Research Center and Mathematical Sciences, \\ University of Southampton, \\ Highfield, Southampton SO17 1BJ, United Kingdom
}

(Received 28 April 2019; published 21 January 2020)

\begin{abstract}
We show that standard puzzles of hot big bang cosmology that motivated the introduction of cosmological inflation, such as the smoothness and horizon problem, the flatness problem, and the relic problem are also solved by holographic models for very early universe based on perturbative three dimensional QFT. In the holographic setup, cosmic evolution is mapped to inverse renormalization group (RG) flow of the dual QFT, and the resolution of the puzzles relies on properties of the RG flow.
\end{abstract}

DOI: 10.1103/PhysRevD.101.021901

\section{INTRODUCTIONS}

The theory of inflation was initially introduced [1-3] as an answer to three problems of hot big bang cosmology: (i) the horizon problem (why is the universe so homogenous despite the fact that separated regions were causally disconnected?), (ii) the flatness problem (why is the Universe as flat as we see it today), and (iii) the relic problem (why we do not see any relics from the very early Universe?). Inflation beautifully resolves these (and other) problems by postulating a period of accelerating expansion in the very early Universe.

What is perhaps the biggest success of this theory is its ability to generate primordial perturbations, which form the seeds for structure formation in the late Universe, and which are in excellent agreement with observations of the cosmic microwave background (CMB) by satellites and other missions. Despite these successes, however, the underlying theory still remains unsatisfactory: it requires fine tuning, there are trans-Planckian issues and questions about initial condition, see for example [4]. The theory of inflation is an effective field theory and we are still lacking a proper understanding of its ultraviolet (UV) completion. This as well as the resolution of the initial singularity require the embedding of inflation in a consistent quantum theory of gravity. Achieving such embedding in string theory is an ongoing effort and the very existence of

\footnotetext{
horatiu.nastase@unesp.br

†.Skenderis@soton.ac.uk
}

Published by the American Physical Society under the terms of the Creative Commons Attribution 4.0 International license. Further distribution of this work must maintain attribution to the author(s) and the published article's title, journal citation, and DOI. Funded by SCOAP. (quasi)-de Sitter solutions in string theory has recently been questioned (see for example [5]). It is thus important to approach this question from different perspectives and explore and further develop alternative models for the very early Universe.

It is widely believed that quantum gravity is holographic [6-8], meaning that there is an equivalent description using a quantum field theory (QFT) (with no gravity) in one dimension less. Holographic dualities are still conjectural and this is even more so in the case of cosmology. The cosmological holographic framework however has already passed a number of nontrivial tests and we will provide additional support in this paper. Work on holographic cosmology was initiated in [9-12], with standard inflation fitting in this framework as a strongly coupled QFT (see for example [13-32]). Holographic cosmology also contains qualitative new models for the very early universe obtained by considering QFTs at weak coupling [33]. These new models correspond to a strongly coupled nongeometric phase of gravity and they will be the focus of this paper.

In the context of cosmology the dual QFT is a three dimensional Euclidean theory, which is located at future infinity and its partition function, in the presence of sources for gauge invariant operators, is identified with the wave function of the universe. The fields parametrizing the (Dirichlet) boundary conditions at future infinity ${ }^{1}$ are

\footnotetext{
${ }^{1}$ Note that the asymptotic structure near the timelike boundary of AdS [34,35] is mapped to the asymptotic structure near the spacelike boundary of de Sitter [36] via analytic continuation [37], see also [38] and [39]. The same analytic continuation also maps general perturbations (at least to quadratic order) around domain-walls/FRW cosmologies [33,40-42], and this translates into specific analytic continuation on the QFT side, as discussed in $[33,40-42]$.
} 
identified with the sources of dual operators and the arguments of the wave function of the Universe [12]. The dimension which is reconstructed holographically is the time direction and cosmic evolution is mapped to inverse RG flow. The holographic description is currently known only for the very early universe, the period usually associated with inflation, and the transition to standard cosmology is via "instant reheating", i.e., the outcome of this period becomes the initial conditions for the subsequent evolution via Einstein equations (see [43]).

In the holographic framework, models are defined by providing the dual QFT, and in the models describing a nongeometric early Universe this is a three dimensional superrenormalizable theory: $S U(N)$ gauge theory for a gauge field $A_{i}$ coupled to scalars $\phi$ and fermions $\psi$, with action [33]

$$
\begin{aligned}
S= & \frac{1}{g_{\mathrm{YM}}^{2}} \int d^{3} x \operatorname{Tr}\left[\frac{1}{2} F_{i j} F^{i j}+(D \phi)^{2}+\bar{\psi} D_{i} \gamma^{i} \psi\right. \\
& \left.+\mu(\bar{\psi} \psi \phi)+\lambda \phi^{4}\right],
\end{aligned}
$$

plus a nonminimal coupling $\int d^{3} x \xi R \phi^{2}$, where $R$ is the scalar curvature- on a flat $3 \mathrm{~d}$ background the nonminimality parameter $\xi$ appears only in the improvement term in the energy momentum tensor. All fields are in the adjoint of $S U(N)$ and we suppress numerical factors and flavor indices (see [44] for the details). This theory has a "generalized conformal structure," which means that if one promotes $g_{\mathrm{YM}}^{2}$ to a field with appropriate conformal transformations the theory becomes conformal $[45,46]$, or that by assigning " $4 \mathrm{~d}$ dimensions" to the fields, $[\Phi]=$ $\left[A_{i}\right]=1,[\psi]=3 / 2$, all terms in the action scale in the same way.

The phenomenology of these models has been worked out in $[33,40-42,47-50]$, using methods from [12,37,51,52]. The models predict a scalar power spectrum of the form

$$
\Delta_{S}^{2}=\frac{\Delta_{0}^{2}}{1+\frac{g q^{*}}{q} \ln \left|\frac{q}{\beta g q^{*}}\right|+\mathcal{O}\left(\frac{g q^{*}}{q}\right)^{2}},
$$

where $\beta, g$ are parameters that are obtained by a 2-loop computation of the 2-point function of the energy momentum tensor and there is a similar form for the tensor power spectrum. These models have been compared against WMAP $[43,53]$ and Planck data $[44,54]$ and it was found that within their regime of validity ${ }^{2}$ they provide an excellent fit to data and are competitive with $\Lambda \mathrm{CDM}$ - the fit to data shows that holographic cosmology $(\mathrm{HC})$ and $\Lambda \mathrm{CDM}$ are within one sigma.

In this paper, we would like to discuss how holographic cosmology addresses the hot Big Bang problems. We will

\footnotetext{
${ }^{2}$ One of the results of $[44,54]$ is that the model becomes nonperturbative at very low multipoles (less than 30) and a nonperturbative evaluation of the power spectrum is needed to model this region.
}

start by first reviewing how inflation solves these problem and then move to discuss them within the context of holographic cosmology. As this part is standard material we will be brief-the details can be found in most cosmology textbooks.

\section{INFLATION AND HOT BIG BANG PROBLEMS}

\section{A. Smoothness and horizon problems, or Why is the Universe uniform and isotropic?}

The question can be formulated as follows: why is the Universe so smooth and correlated on large scales when different parts of the sky were not in causal contact at the initial time? In hot big bang cosmology, the points in the CMB separated by more than $1.6^{\circ}$ could not have been in causal contact because their past light cones do not overlap before the spacetime is terminated by the initial singularity (see for example [55,56]). One has to increase the horizon distance at the surface of last scattering at least by a factor of 66 to be consistent with observations.

Inflation's answer to this problem is that the exponential blow up of a small patch creates the whole observable Universe, and this patch was in causal contact. Let $t_{\mathrm{bi}}$ the time inflation began, $t_{I}$ it ended and $N_{e}=H\left(t_{I}\right)\left(t_{I}-t_{\mathrm{bi}}\right)$ the number of e-foldings. Assuming nothing much happened between the end of inflation and the beginning of radiation domination, a short computation shows that the horizon problem is avoided provided we have enough efoldings of inflation.

\section{B. Flatness problem, or Why do we have $\Omega \simeq 1$ in the past?}

Observations tell us that the Universe is approximately flat today. If the Universe were exactly flat in the past, then cosmic evolution would preserve this property and it would be exactly flat today. However, if $\Omega-1 \neq 0$ but small, extrapolating into the past using matter domination (MD) and radiation domination (RD) formulae, we find an extremely flat Universe at initial times. Quantitatively, $\Omega(t)-1 \propto t^{2(1-p)}$ for $a(t) \propto t^{p}$. In both RD $(p=1 / 2)$ and $\operatorname{MD}(p=2 / 3)$ eras, $\Omega(t)-1$ increases with time, so it must have been very small in the past, and to avoid fine tuning we need a period of $p>1$, to bring it down to the value we obtain now.

Indeed, inflation naturally drives $\Omega$ very close to one. A short computation (see for example [55]) shows that $\Omega_{0}-1=\left(\Omega\left(t_{b i}\right)-1\right) e^{-2 N_{e}}\left(\left(a\left(t_{I}\right) H\left(t_{I}\right) /\left(a_{0} H_{0}\right)\right)^{2}\right.$, where (as usual) the subscript 0 denotes todays values, and this leads to exactly the same condition needed to solve the horizon problem.

\section{Relic (monopole) problem, or Why do we not see relics in the Universe?}

In phase transitions we would obtain relics, for example monopoles from grand unified theories (GUT) phase 
transitions, where we would expect about one monopole per nucleon, or $10^{-9}$ monopoles per photon. However, from direct searches in materials on Earth we know that there are $\leq 10^{-30}$ monopoles per nucleon (see [55], chapter 4.1.C), so we need a reduction factor of $10^{-30}$.

Relics in general are also constrained by their gravitational effects (see [57], chapter 7.5): in order to not overclose the Universe, we need a reduction factor of about $10^{-11}$, much less stringent than for monopoles.

Inflation's answer to the problem of both monopoles and general relics is that it dilutes them during the period of exponential inflation. Inflation therefore needs to happen after, or at most during the phase transition.

\section{RESOLUTION USING HOLOGRAPHIC COSMOLOGY}

We now turn to the same questions in the context of holographic cosmology, where gravity is strongly coupled and the dual field theory is weakly coupled.

\section{A. Smoothness and horizon problems}

These models describe a nongeometric early Universe so geometric concepts such as light-cones are meaningless, and the traditional formulation of the problem is not valid. Nevertheless it would be useful to understand the mechanism that put in causal contact parts of the sky that from the perspective of hot big bang cosmology appear to be uncorrelated.

In holographic cosmology cosmological observables are computed from correlation functions of the dual QFT, and the correlations at the surface of last scattering are those of these correlators. In QFT correlation functions at different scales are related to each other via renormalization group flow. As time evolution is mapped to inverse RG flow, points widely separated at the surface of last scattering would be linked by RG flows that connect the UV with the deep IR, so as long as the QFT is well-defined in the IR, there is no horizon problem, as any two points at the surface of scattering will be causally linked via a deep enough RG flow.

The theories we discuss here are superrenormalizable so they are naively IR divergent. This is the holographic dual of the bulk initial singularity. These theories however are expected to be nonperturbatively IR finite $[58,59]$ and this has recently been confirmed by lattice studies [60]. It follows that in this class of models there is no horizon problem, irrespectively of the details of each model.

We now illustrate that the usual inflationary resolution of the horizon problem is an example of the same mechanism. For concreteness we discuss the case of asymptotically de Sitter inflation but the same comments apply more generally. Any two points separated by distance $L$ at the spacelike boundary of de Sitter (the end of this phase) may be linked via bulk geodesics that go to the interior of de Sitter.
From the perspective of the dual QFT (and after using the domain-wall/cosmology correspondence [61] to map this question to $\mathrm{AdS}$ ), the (renormalized) length of these geodesics provide the 2-point function of a dual operator inserted at each of the two points [62]. A short computation (see for example [63,64]) shows that $L \propto 1 / r_{0}$, where $r_{0}$ is the maximum radial distance reached in the bulk. Recall that the radial coordinate encodes RG flow in the dual QFT, so the number e-folding corresponds to the amount of RG flow for which the dual field theory is strongly coupled and nearly conformal: it is simple to verify (using the fact that $\left.L \propto 1 / r_{0}\right)$ that the factor multiplying the RG scale corresponds to the factor $e^{N_{e}}$ in inflation.

\section{B. Flatness problem}

To formulate the question in the context of holographic cosmology, we consider a small deviation from a flat background $(\Omega=1)$ and we would like to show that under time evolution (=inverse RG flow) the flat geometry is an attractor. Like in the inflationary case, this needs to be addressed independently of the usual cosmology that follows: we must show that the nongeometric phase alone can do this.

In holography the spacetime where the dual QFT lives is a fixed nondynamical background, so one may wonder whether the flatness problem makes sense in this context. A small deviation from flatness means that the spacetime metric is $g_{i j}=\delta_{i j}+h_{i j}$, where $\delta_{i j}$ is the metric on flat $\mathbb{R}^{3}$ and $h_{i j}$ is a small deviation. By a standard argument, the deviation induces a new coupling in the action $\int d^{3} x T^{i j} h_{i j}$, where $T_{i j}$ is the energy-momentum tensor of the dual QFT on $\mathbb{R}^{3}$ (plus higher order terms). The new coupling $h_{i j}$ will run under RG flow and (as time evolution is inverse RG flow) this is the counterpart of the fact that the density parameter $\Omega$ evolves in a nonflat Friedmann-LemaîtreRobertson-Walker. Note that if $h_{i j}=0$, this coupling will not be induced by the RG flow (in a Lorentz invariant QFT) and this is counterpart of the statement that if the Universe is flat, it remains flat at all times.

The flatness question is now whether the new coupling dies off or dominates in the UV. If it dies off then the flat geometry is an attractor, as in inflation. The perturbative superrenormalizable QFTs [with action given in (1)] that feature in holographic cosmology have a generalized conformal structure and this implies that when the coupling is very small they effectively behave like CFTs: they are nearly conformal. Since we are interested in the late time behavior and the QFT is superrenormalizable, there is no loss of generality to assume that we are in the regime where the QFT is nearly conformal. The question is then whether the deforming operator (i.e., $T^{i j}$ ) is relevant or irrelevant. Since the deformation is also assumed to be very small in the UV $\left(\Omega \sim 10^{-54}\right)$, it suffices to compute the dimension of 
$T$ in the undeformed theory. ${ }^{3}$ If the operator is relevant it would die off in the UV and dominate in the IR and the opposite if it is irrelevant.

We therefore need to determine the dimension of $T$ and this can be done from its 2-point function. In momentum space (and close to the fixed point) the 2-point function should behave as $q^{2 \Delta-d}$ (see for example [65]) and we can extract $\Delta$ from it. $T_{i j}$ is of course marginal (dimension 3 in 3 dimensions) at the classical level, and at the quantum level the $\left\langle T_{i j} T_{k l}\right\rangle$ correlator decomposes into a scalar and a tensor piece, both of the type $q^{3} N^{2} f\left(g_{\text {eff }}^{2}\right)$, where $g_{\text {eff }}^{2}=$ $g^{2} N / q$ is dimensionless. The factor of $q^{3}$ captures the classical dimension of $T$ and implies that to leading order the CMBR power spectra are scale invariant. In perturbation theory, $g_{\mathrm{eff}}^{2} \ll 1$, and at 2-loops (see [40,43,54] for details) the form $f$ is ${ }^{4}$

$$
f\left(g_{\mathrm{eff}}^{2}\right)=f_{0}\left(1-f_{1} g_{\mathrm{eff}}^{2} \ln g_{\mathrm{eff}}^{2}+f_{2} g_{\mathrm{eff}}^{2}+\mathcal{O}\left(g_{\mathrm{eff}}^{2}\right)\right),
$$

where $f_{1}<0$ both for the best fit to the CMBR data, and for most of the general theoretical parameter space. This implies (again for $g_{\text {eff }}^{2} \ll 1$ ) that $f\left(g_{\text {eff }}^{2}\right) \propto q^{2 \delta} \sim$ $1-2 \delta \ln g_{\text {eff }}^{2}+\cdots$ giving $2 \delta \simeq f_{1} g_{\text {eff }}^{2}<0$, and thus $\Delta=$ $3+\delta$ making $T_{\mu \nu}$ (marginally) relevant. ${ }^{5}$ This means that the perturbation will die off in the UV and it would lead to changes of order one in the IR. Recalling that in holographic cosmology time evolution corresponds to inverse RG flow, i.e. from IR to UV, this is precisely what we set out to show.

\section{Relic and monopole problem}

Let us start with monopoles. To study this problem our starting point should be a bulk theory with GUT phase transition and analyse how the effects of monopoles are encoded in the dual QFT. To avoid the monopole problem we need to establish that their effects are washed out at late times, or equivalently in the UV from the perspective of the dual QFT.

Bulk gauge symmetries correspond to boundary global symmetries, so to properly analyze this problem we would need to consider boundary QFTs that have the required global symmetry and pattern of symmetry breaking. It is an interesting problem (that we leave for future work) to

\footnotetext{
${ }^{3}$ In the deformed theory, the leading correction can be computed using conformal perturbation theory and it is of order $\mathcal{O}\left(h_{i j}^{2}\right)$.

${ }^{4}$ Note that as the theory (1) is asymptotically free, the two point function $\left\langle T_{i j} T_{k l}\right\rangle$ in the undeformed theory approaches its freefield value as $q \rightarrow \infty$ (and thus $g_{\text {eff }}^{2} \rightarrow 0$ ), i.e., all loop corrections vanish. Here we are interested to extract the precise way these corrections go to zero, as this controls how the new coupling behaves in the deformed theory.

${ }^{5}$ Note that in the standard CMBR inflationary description, $f_{1}<0$ translates into a red tilt $\left(n_{s}-1<0\right)$. Turning things around flatness implies that the spectrum should be red.
}

classify the QFTs with such properties, start with 't HooftPolyakov monopoles in the bulk and analyse their effects in complete generality.

Here we will proceed by solving a related problem: we will consider instead a Dirac monopole in the bulk. The bulk theory will thus involve a $U(1)$ gauge field and we should consider a monopole field $A_{\mu}$ in the bulk, which by the standard AdS/CFT dictionary, will induce a new coupling in the boundary theory, $\int d^{3} x A_{(0) i} \tilde{j}^{i}$, where $\tilde{j}^{i}$ is the magnetic current and $A_{(0) i}$ is the boundary value of $A_{\mu}$. As in our study of the flatness problem, we would like to study whether such a coupling will have an effect in the $\mathrm{UV}$, and this can be analyzed by extracting the dimension of $\tilde{j}^{i}$ near the UV when the theory is nearly conformal.

This is still a nontrivial problem as we usually work with electric variables. Luckily, 3d CFTs with a global $U(1)$ symmetry allow for an $S l(2 ; \mathbb{Z})$ action, whose $S$-generator exchanges the electric and magnetic currents [66]. In the bulk this operations corresponds to usual electromagnetic duality (see also $[67,68]$ ). The 2-point function of symmetry currents in a CFT is given by (ignoring the contact terms which are relevant in general for the action of $S l(2 ; \mathbb{Z})$ but not relevant for us)

$$
\left\langle j_{i}(q) j_{j}(-q)\right\rangle \simeq q\left(\delta_{\mu \nu}-\frac{q_{\mu} q_{\nu}}{q^{2}}\right) t
$$

where $t$ is a constant (in a CFT) and the $S$-generator takes $t \rightarrow 1 / t$. This is not a symmetry: it maps one CFT to another. In a theory with a generalized conformal structure the form of the 2-point function is the same but $t$ is now a function of $g_{\text {eff }}^{2}$. We will assume that the discussion in [66] generalizes to such theories, at least when $g_{\text {eff }} \ll 1$ and the theory is nearly free (and thus nearly conformal).

Our strategy is now to start from a theory with an electric current, compute its 2-point function to 2-loop order and then use the $S$-operation to obtain the corresponding result for the theory with the magnetic current, from which we will read off its anomalous dimension. This computation will be done in a toy model: an $S U(N)$ gauge theory for a gauge field $A_{i}$ coupled to 6 complex scalars $\phi_{\alpha}^{a}, a=1,2,3$ and $\alpha=1,2$, with the index $a$ transforming in the 3 of $S O(3)$ [all fields are also in the adjoint of $S U(N)$ ]. The Euclidean action is (we denote spatial indices by $i=1,2,3$ )

$S=\frac{2}{g_{\mathrm{YM}}^{2}} \int d^{3} x \operatorname{Tr}\left[\frac{1}{4} F_{i j} F^{i j}+\left|D_{i} \vec{\phi}_{\alpha}\right|^{2}+\lambda\left|\vec{\phi}_{1} \times \vec{\phi}_{2}\right|^{2}\right]$

and the global symmetry current is $j_{i}^{a}=\sum_{\alpha=1,2} \vec{\phi}_{\alpha}^{*} T^{a} D_{i} \vec{\phi}_{\alpha}+$ H.c., where $T_{a}$ are $S O(3)$ generators. This model has the feature of admitting Abelian vortex solutions of the form $\phi_{1}^{a}=\phi_{1}^{a}(r) e^{i \phi}, \phi_{2}^{a}=\phi_{2}^{a}(r) e^{i \phi}$, which may be used to justify the $S$-operation below, as it will be explained in detail elsewhere [69]. 
A 2-loop calculation, whose details will be presented in [69], leads to the 2-point function in (4) with $t=1+2 g_{\text {eff }}^{2} / \pi^{2} \ln q$, which means that the anomalous dimension of $j_{i}^{a}$ is given by $2 \delta(j)=\frac{4}{\pi^{2}} g_{\text {eff }}^{2}>0$, making $j_{\mu}^{a}$ an irrelevant operator. Applying the $S$-operation then implies $\delta(\tilde{j})=-\delta(j)=-\frac{2}{\pi^{2}} g_{\text {eff }}^{2}<0$. It follows that the effects of the Dirac monopole in the bulk are washed out in the UV.

In general such analysis may be used to rule out holographic models: only models with negative anomalous dimension for the magnetic current solve the monopole problem.

Other relics may be studied in a similar way. We note however that the main effect is via the gravitational perturbation they generate and as such analysis will be similar to that of the flatness problem.

\section{ENTROPY PROBLEM AND THE ARROW OF TIME}

The current total entropy of the Universe (about $10^{88}$ per horizon volume today) requires an explanation because it is appears either too large or too low. Evolving to the past with standard RD and MD formulas, we find that the entropy inside the horizon at big bang nucleosynthesis was $S_{H}\left(t_{\mathrm{BBN}}\right) \sim 10^{63}$, but one may have expected a number of order one per horizon in standard cosmology, at least at the end of a phase transition. On the hand, as emphasized by Penrose [70] (see also [71,72]) the entropy of the observable Universe could have been a lot higher: if the entire mass of the observable universe were collected into a single black hole the entropy would be about $10^{121}$. Usually this version is associated with the question of the arrow of time and the very special nature of the initial conditions needed in the very early Universe, and in general this issue is considered an open problem.

In holography, time evolution is inverse RG flow, so the arrow of time is linked to the monotonicity of the RG flow, which for three dimensional QFTs was established in [73]. The total entropy grows because the degrees of freedom in the UV are larger than that in the IR. This is a general property of RG flows and not a choice of a model. Furthermore, universality of IR dynamics makes the low entropic initial conditions natural. To explain quantitatively why the total entropy is as large we observe it today requires developing a holographic model for reheating and this is outside the scope of this work.

\section{CONCLUSIONS}

In this paper we have shown that the (nongeometric) holographic cosmology model of [33] is capable of solving the standard problems of hot big bang cosmology: the smoothness and horizon problems, the flatness problem and the monopole and relic problems. In holographic cosmology time evolution translates into inverse RG flow and these problems are naturally resolved using properties of the RG flow. In these models the resolution of the initial singularity is mapped to the IR finiteness of the dual QFT and the arrow of time is linked with the monotonicity of RG flow. Together with the previously found fact that the CMBR fitting is as good as for standard $\Lambda$ CDM with inflation our results mean that holographic cosmology is a viable alternative for a Standard Model of cosmology.

\section{ACKNOWLEDGMENTS}

We would like to thank Juan Maldacena and Paul McFadden for useful discussions. K. S. would like to thank the ICTP-SAIFR and USP for hospitality during the initial stage of this work, and acknowledges support from FAPESP Grants No. 2016/01343-7, and No. 2014/ 18634-9. This project has received funding/support from the European Union's Horizon 2020 research and innovation programme under the Marie Sklodowska-Curie Grant agreement No. 690575. KS is also supported in part by the Science and Technology Facilities Council (Consolidated Grant "Exploring the Limits of the Standard Model and Beyond"). The work of $\mathrm{H}$. N. is supported in part by CNPq Grant No. 304006/2016-5 and FAPESP grant 2014/18634-9. H. N. would also like to thank the ICTP-SAIFR for their support through FAPESP grant 2016/01343-7.
[1] A. H. Guth, The inflationary universe: A possible solution to the horizon and flatness problems, Phys. Rev. D 23, 347 (1981); Adv. Ser. Astrophys. Cosmol. 3, 139 (1987).

[2] A. D. Linde, A new inflationary universe scenario: A possible solution of the horizon, flatness, homogeneity, isotropy and primordial monopole problems, Phys. Lett. 108B, 389 (1982); Adv. Ser. Astrophys. Cosmol. 3, 149 (1987).
[3] A. Albrecht and P. J. Steinhardt, Cosmology for Grand Unified Theories with Radiatively Induced Symmetry Breaking, Phys. Rev. Lett. 48, 1220 (1982); Adv. Ser. Astrophys. Cosmol. 3, 158 (1987).

[4] R. H. Brandenberger, Inflationary cosmology: Progress and problems, arXiv:hep-ph/9910410.

[5] G. Obied, H. Ooguri, L. Spodyneiko, and C. Vafa, De Sitter space and the swampland, arXiv:1806.08362. 
[6] G. 't Hooft, Dimensional reduction in quantum gravity, Conf. Proc. C 930308, 284 (1993).

[7] L. Susskind, The world as a hologram, J. Math. Phys. (N.Y.) 36, 6377 (1995).

[8] J. M. Maldacena, The Large $N$ limit of superconformal field theories and supergravity, Int. J. Theor. Phys. 38, 1113 (1999); Adv. Theor. Math. Phys. 2, 231 (1998).

[9] E. Witten, Quantum gravity in de Sitter space, arXiv:hep-th/ 0106109.

[10] A. Strominger, The dS / CFT correspondence, J. High Energy Phys. 10 (2001) 034.

[11] A. Strominger, Inflation and the dS / CFT correspondence, J. High Energy Phys. 11 (2001) 049.

[12] J. M. Maldacena, Non-Gaussian features of primordial fluctuations in single field inflationary models, J. High Energy Phys. 05 (2003) 013.

[13] J. M. Maldacena and G. L. Pimentel, On graviton nonGaussianities during inflation, J. High Energy Phys. 09 (2011) 045.

[14] J. B. Hartle, S. W. Hawking, and T. Hertog, Accelerated expansion from negative $\Lambda$, arXiv:1205.3807.

[15] J. B. Hartle, S. W. Hawking, and T. Hertog, Quantum probabilities for inflation from holography, J. Cosmol. Astropart. Phys. 01 (2014) 015.

[16] K. Schalm, G. Shiu, and T. van der Aalst, Consistency condition for inflation from (broken) conformal symmetry, J. Cosmol. Astropart. Phys. 03 (2013) 005.

[17] A. Bzowski, P. McFadden, and K. Skenderis, Holography for inflation using conformal perturbation theory, J. High Energy Phys. 04 (2013) 047.

[18] I. Mata, S. Raju, and S. Trivedi, CMB from CFT, J. High Energy Phys. 07 (2013) 015.

[19] J. Garriga and Y. Urakawa, Inflation and deformation of conformal field theory, J. Cosmol. Astropart. Phys. 07 (2013) 033.

[20] P. McFadden, On the power spectrum of inflationary cosmologies dual to a deformed CFT, J. High Energy Phys. 10 (2013) 071.

[21] A. Ghosh, N. Kundu, S. Raju, and S. P. Trivedi, Conformal invariance and the four point scalar correlator in slow-roll inflation, J. High Energy Phys. 07 (2014) 011.

[22] J. Garriga and Y. Urakawa, Holographic inflation and the conservation of $\zeta$, J. High Energy Phys. 06 (2014) 086.

[23] N. Kundu, A. Shukla, and S. P. Trivedi, Constraints from conformal symmetry on the three point scalar correlator in inflation, J. High Energy Phys. 04 (2015) 061.

[24] J. Garriga, K. Skenderis, and Y. Urakawa, Multi-field inflation from holography, J. Cosmol. Astropart. Phys. 01 (2015) 028.

[25] P. McFadden, Soft limits in holographic cosmology, J. High Energy Phys. 02 (2015) 053.

[26] N. Arkani-Hamed and J. Maldacena, Cosmological collider physics, arXiv:1503.08043.

[27] N. Kundu, A. Shukla, and S. P. Trivedi, Ward identities for scale and special conformal transformations in inflation, J. High Energy Phys. 01 (2016) 046.

[28] T. Hertog and E. van der Woerd, Primordial fluctuations from complex AdS saddle points, J. Cosmol. Astropart. Phys. 02 (2016) 010.
[29] J. Garriga, Y. Urakawa, and F. Vernizzi, $\delta N$ formalism from superpotential and holography, J. Cosmol. Astropart. Phys. 02 (2016) 036.

[30] J. Garriga and Y. Urakawa, Consistency relations and conservation of $\zeta$ in holographic inflation, J. Cosmol. Astropart. Phys. 10 (2016) 030.

[31] S. W. Hawking and T. Hertog, A smooth exit from eternal inflation?, J. High Energy Phys. 04 (2018) 147.

[32] N. Arkani-Hamed, D. Baumann, H. Lee, and G. L. Pimentel, The cosmological bootstrap: Inflationary correlators from symmetries and singularities, arXiv:1811.00024.

[33] P. McFadden and K. Skenderis, Holography for cosmology, Phys. Rev. D 81, 021301 (2010).

[34] C. Fefferman and C. R. Graham, Conformal invariants, in Elie Cartan et les Mathématiques d'aujourd'hui (Astérisque, Paris, 1985), p. 95.

[35] S. de Haro, S. N. Solodukhin, and K. Skenderis, Holographic reconstruction of space-time and renormalization in the AdS/CFT correspondence, Commun. Math. Phys. 217, 595 (2001).

[36] A. A. Starobinsky, Isotropization of arbitrary cosmological expansion given an effective cosmological constant, JETP Lett. 37, 66 (1983) [http://www.jetpletters.ac.ru/ps/1488/ article_22720.shtml].

[37] K. Skenderis, Lecture notes on holographic renormalization, Classical Quantum Gravity 19, 5849 (2002).

[38] P. O. Mazur and E. Mottola, Weyl cohomology and the effective action for conformal anomalies, Phys. Rev. D 64, 104022 (2001).

[39] A. Poole, K. Skenderis, and M. Taylor, (A) $\mathrm{dS}_{4}$ in Bondi gauge, Classical Quantum Gravity 36, 095005 (2019).

[40] P. McFadden and K. Skenderis, The holographic universe, J. Phys. 222, 012007 (2010).

[41] P. McFadden and K. Skenderis, Holographic nonGaussianity, J. Cosmol. Astropart. Phys. 05 (2011) 013.

[42] P. McFadden and K. Skenderis, Cosmological 3-point correlators from holography, J. Cosmol. Astropart. Phys. 06 (2011) 030.

[43] R. Easther, R. Flauger, P. McFadden, and K. Skenderis, Constraining holographic inflation with WMAP, J. Cosmol. Astropart. Phys. 09 (2011) 030.

[44] N. Afshordi, C. Coriano, L. Delle Rose, E. Gould, and K. Skenderis, From Planck Data to Planck Era: Observational Tests of Holographic Cosmology, Phys. Rev. Lett. 118, 041301 (2017).

[45] A. Jevicki and T. Yoneya, Space-time uncertainty principle and conformal symmetry in $D$ particle dynamics, Nucl. Phys. B535, 335 (1998); A. Jevicki, Y. Kazama, and T. Yoneya, Generalized conformal symmetry in $D$-brane matrix models, Phys. Rev. D 59, 066001 (1999).

[46] I. Kanitscheider, K. Skenderis, and M. Taylor, Precision holography for non-conformal branes, J. High Energy Phys. 09 (2008) 094.

[47] A. Bzowski, P. McFadden, and K. Skenderis, Holographic predictions for cosmological 3-point functions, J. High Energy Phys. 03 (2012) 091.

[48] C. Coriano, L. Delle Rose, and M. Serino, Three and four point functions of stress energy tensors in $D=3$ for the analysis of cosmological non-Gaussianities, J. High Energy Phys. 12 (2012) 090. 
[49] S. Kawai and Y. Nakayama, Improvement of energymomentum tensor and non-Gaussianities in holographic cosmology, J. High Energy Phys. 06 (2014) 052.

[50] P. McFadden and K. Skenderis, Observational signatures of holographic models of inflation, arXiv:1010.0244.

[51] I. Papadimitriou and K. Skenderis, AdS/CFT correspondence and geometry, IRMA Lect. Math. Theor. Phys. 8, 73 (2005).

[52] I. Papadimitriou and K. Skenderis, Correlation functions in holographic RG flows, J. High Energy Phys. 10 (2004) 075.

[53] M. Dias, Cosmology at the boundary of de Sitter using the dS/QFT correspondence, Phys. Rev. D 84, 023512 (2011).

[54] N. Afshordi, E. Gould, and K. Skenderis, Constraining holographic cosmology using Planck data, Phys. Rev. D 95, 123505 (2017).

[55] S. Weinberg, Cosmology (Oxford University Press, New York, 2008), p. 593.

[56] D. Baumann and L. McAllister, Inflation and string theory, arXiv:1404.2601.

[57] E. W. Kolb and M. S. Turner, The early universe, Front. Phys. 69, 1 (1990).

[58] R. Jackiw and S. Templeton, How superrenormalizable interactions cure their infrared divergences, Phys. Rev. D 23, 2291 (1981).

[59] T. Appelquist and R. D. Pisarski, High-temperature YangMills theories and three-dimensional quantum chromodynamics, Phys. Rev. D 23, 2305 (1981).

[60] J. K. L. Lee, L. Del Debbio, A. Jüttner, A. Portelli, and K. Skenderis, Towards a holographic description of cosmology: Renormalisation of the energy-momentum tensor of the dual QFT, arXiv:1909.13867.

[61] K. Skenderis and P. K. Townsend, Hidden Supersymmetry of Domain Walls and Cosmologies, Phys. Rev. Lett. 96, 191301 (2006).
[62] C. R. Graham and E. Witten, Conformal anomaly of submanifold observables in AdS/CFT correspondence, Nucl. Phys. B546, 52 (1999).

[63] J. M. Maldacena, Wilson Loops in Large $N$ Field Theories, Phys. Rev. Lett. 80, 4859 (1998).

[64] S. J. Rey and J. T. Yee, Macroscopic strings as heavy quarks in large $N$ gauge theory and anti-de Sitter supergravity, Eur. Phys. J. C 22, 379 (2001).

[65] A. Bzowski, P. McFadden, and K. Skenderis, Implications of conformal invariance in momentum space, J. High Energy Phys. 03 (2014) 111.

[66] E. Witten, SL(2,Z) action on three-dimensional conformal field theories with Abelian symmetry, in From Fields to Strings, edited by M. Shifman et al., Vol. 2 (World Scientific, Singapore, 2005), pp. 1173-1200, https://doi .org/10.1142/5621.

[67] C. P. Herzog, P. Kovtun, S. Sachdev, and D. T. Son, Quantum critical transport, duality, and M-theory, Phys. Rev. D 75, 085020 (2007).

[68] J. Murugan, H. Nastase, N. Rughoonauth, and J. P. Shock, Particle-vortex and Maxwell duality in the $\mathrm{AdS}_{4} \times$ $\mathbb{C P}^{3} / \mathrm{ABJM}$ correspondence, J. High Energy Phys. 10 (2014) 51.

[69] H. Nastase and K. Skenderis (to be published).

[70] R. Penrose, Singularities and Time-Asymmetry, in General Relativity, an Einstein Centenary Survey, edited by S. W. Hawking and W. Israel (Cambridge University Press, Cambridge, England, 1979).

[71] S. M. Carroll and J. Chen, Spontaneous inflation and the origin of the arrow of time, arXiv:hep-th/0410270.

[72] R. M. Wald, The Arrow of time and the initial conditions of the universe, Stud. Hist. Phil. Mod. Phys. 37, 394 (2006).

[73] H. Casini and M. Huerta, On the RG running of the entanglement entropy of a circle, Phys. Rev. D 85, 125016 (2012). 